\title{
Fuzzy Distance between Fuzzy Values
}

\section{Qihao CHEN $^{* 1}$ Shin KAWASE*2}

\section{Introduction}

Fuzzy value is a Fuzzy set on $[0,1]$. For the definition, see section 2. Fuzzy values have originated from Fuzzy truth values[4], and find several applications in divers realms, e.g., Fuzzy controls, Fuzzy reasonings, inverse problems of Fuzzy relations, and others.

Engineers often use triangular Fuzzy sets, which are Fuzzy values of special kind. Even when inputs are triangular ones, outputs we will obtain are not necessarily so. Hence it would be significant, for further practical as well as theoretical developments, to investigate Fuzzy values in general.

To promote investigation of Fuzzy values, especially of their topological properties, we introduce here Fuzzy distance between Fuzzy values.

We often make use of distance between two points, which is a nonnegative real number, but its measurement is accompanied by some errors. Fuzzy distance which we introduce here is itself a Fuzzy value, and may be considered more suitable for practical purpose. Applications of Fuzzy distance would be our further subjects.

According to customary usage, we denote by $[a$, $b]$ a closed interval on the real line , which implies of course that $a \leqq b$ and $[a, b] \neq \emptyset$.

\section{Definition and Fundamental Prop- erties of Fuzzy Values}

A map $A:[0,1] \rightarrow[0,1]$ is said a Fuzzy set in the closed interval $[0,1]$. The value $A(x)$ is called the membership function of the Fuzzy set $A$. The set of Fuzzy sets in $[0,1]$ is denoted as $\mathfrak{F}_{1}[0,1]$.

Let $A \in \mathfrak{F}_{1}[0,1]$. For $\alpha \in[0,1]$ we put

$A_{\alpha}= \begin{cases}\{x \in[0,1] \mid A(x) \geqq \alpha\}, & \text { if } 0<\alpha \leqq 1, \\ \{x \in[0,1] \mid A(x)>0\}, & \text { if } \alpha=0 .\end{cases}$

$A_{\alpha}$ is said the $\alpha$-cut of the Fuzzy set $A$.

Definition 2.1 $A \in \mathfrak{F}_{1}[0,1]$ is called a Fuzzy value

*1 Beijing University of Post and Telecommunications

*2 Teikyo Heisei University

帝宗中成大学情城兴部情報システム兴科 if $A_{\alpha}$ are closed intervals for any $\alpha \in[0,1]$. Usu ally we write $A_{\alpha}=\left[a_{-}(\alpha), a_{+}(\alpha)\right]$. The set of Fuzzy values is denoted as $\mathfrak{M}[0,1]$.

The following theorems, proved in [1], are very useful in this note:

Theorem 2.1 Let $A \in \mathfrak{M}[0,1]$. Write $A_{\alpha}=$ $\left[a_{-}(\alpha), a_{+}(\alpha)\right]$. Then we have

$\left(1_{1}\right)$ If $\alpha_{1} \leqq \alpha_{2}$, then $A_{\alpha_{2}} \subseteq A_{\alpha_{1}}$, that is, $a_{-}\left(\alpha_{1}\right) \leqq$ a. $\left(\alpha_{2}\right), a_{+}\left(\alpha_{1}\right) \geqq a_{+}\left(\alpha_{2}\right)$.

$\left(1_{2}\right)$ Both $a_{-}(\alpha)$ and $a_{+}(\alpha)$ are left continuous on $(0,1]$.

$\left(1_{3}\right)$ Both $a(\alpha)$ and $a_{+}(\alpha)$ are right continuous at $\alpha=0$.

Theorem 2.2 Let $\left\{U(\alpha)=\left[u_{-}(\alpha), u_{+}(\alpha)\right] \mid 0 \leqq\right.$ $\alpha \leqq 1\}$ be a (non-empty) family of closed intervals. If $U(\alpha)$ satisfies the following conditions $\left(2_{1}\right)-$ $\left(2_{3}\right)$, then there exists uniquely a Fuzzy value $A$ such that $A_{\alpha}=U(\alpha), \alpha \in[0,1]$.

$\left(2_{1}\right)$ If $\alpha_{1} \leqq \alpha_{2}$, then $U\left(\alpha_{2}\right) \subseteq U\left(\alpha_{1}\right)$.

$\left(2_{2}\right)$ Both $u_{-}(\alpha)$ and $u_{+}(\alpha)$ are left continuous on $(0,1]$.

$\left(2_{3}\right)$ Both $u_{-}(\alpha)$ and $u_{+}(\alpha)$ are right continuous at $\alpha=0$.

\section{Crisp distance between Fuzzy val- ues}

Definition 3.1 A map $d: \mathfrak{M}[0,1]^{2} \rightarrow \quad$ is said Crisp distance on $\mathfrak{M}[0,1]$ if for any $A, B, C \in \mathfrak{M}$ $[0,1]$

1. $d(A, B) \geqq 0 ; d(A, B)=0 \Leftrightarrow A=B$;

2. $d(A, B)=d(B, A)$;

3. $d(A, B) \leqq d(A, C)+d(C, B)$.

We will use the following crisp distance of the special type (see also [2]) :

(3.1) $D(A, B)=\sup _{\alpha \in[0,1]} d_{H}\left(A_{\alpha}, B_{\alpha}\right)$

for $A, B \in \mathfrak{M}[0,1]$,

where $d_{H}(E, F)$ is the Hausdorff distance between two compact sets $\mathrm{E}, \mathrm{F} \subset$ : 


$$
d_{H}(E, F)=\max \left(\sup _{y \in F \in E} \inf _{y}|x-y|, \sup _{x \in E, y \in F}|x-y|\right) .
$$

Since $A, B \in \mathfrak{M}[0,1]$, we can write as $A_{\alpha}=\left[a_{-}(\alpha)\right.$, $\left.a_{+}(\alpha)\right], B=\left[b_{-}(\alpha), b_{+}(\alpha)\right]$. Hence we have

$$
\begin{array}{r}
D(A, B)=\sup _{\alpha=0,1 \mid} \max \left(\left|a_{-}(\alpha)-b_{-}(\alpha)\right|,\right. \\
\left.\left|a_{+}(\alpha)-b_{+}(\alpha)\right|\right) .
\end{array}
$$

\section{Addition of Fuzzy values}

Definition 4.1 Let $A, B \in \mathbb{M}[0,1]$ and $A_{\alpha}=$ $\left[a_{-}(\alpha), a_{+}(\alpha)\right], B_{\alpha}=\left[b_{-}(\alpha), b_{+}(\alpha)\right]$. We put

(4.1) $V(\alpha)=\left[\min \left\{a_{-}(\alpha)+b_{-}(\alpha), 1\right\}\right.$, $\left.\min \left\{a_{+}(\alpha)+b_{+}(\alpha), 1\right\}\right]$.

Then, as shown in Theorem 4.1 below, the family $\{V(\alpha) \mid \alpha \in[0,1]\}$ of closed intervals determines a Fuzzy value which we take as the sum $A \oplus B$ of $A$, $B$ :

\section{$A \oplus B=$ the Fuzzy value determined by $\{V(\alpha) \mid \alpha \in[0,1]\}$ in (4.1).}

Theorem 4.1 The family $\{V(\alpha) \mid \alpha \in[0,1]\}$ in (4.1) satisfies the conditions $\left(2_{1}\right)-\left(2_{3}\right)$ in Theorem 2.2. Hence it defines a Fuzzy value.

Proof. Put $u_{-}(\alpha)=\min \left\{a_{-}(\alpha)+b(\alpha), 1\right\}, u_{+}(\alpha)=$ $\min \left\{a_{+}(\alpha)+b_{+}(\alpha), 1\right\}$.

$\left(2_{1}\right)$ For $0 \leqq \alpha_{1} \leqq \alpha_{2} \leqq 1$ we have obviously

$$
\begin{aligned}
& u\left(\alpha_{1}\right) \leqq u_{+}\left(\alpha_{2}\right), u_{+}\left(\alpha_{1}\right) \\
& \geq u_{+}\left(\alpha_{2}\right), \text { hence } V\left(\alpha_{2}\right) \subseteq V\left(\alpha_{1}\right) .
\end{aligned}
$$

$\left(2_{2}\right)$ For $\alpha \in(0,1]$ we have

$$
\begin{aligned}
\lim _{\beta} & \min \left\{a_{ \pm}(\beta)+b_{ \pm}(\beta), 1\right\} \\
& =\min \left\{\lim _{\beta}(\alpha-0)\right. \\
& \left.=\min \left\{a_{ \pm}(\beta)+b_{ \pm}(\beta)\right), 1\right\} \\
& \left.=b_{ \pm}(\alpha), 1\right\} .
\end{aligned}
$$

$\left(2_{3}\right)$ We have

$$
\begin{aligned}
\lim _{\beta},-0 & \min \left\{a_{ \pm}(\beta)+b_{ \pm}(\beta), 1\right\} \\
& =\min \left\{\lim _{\beta}\left(a_{ \pm}(\beta)+b_{ \pm}(\beta)\right), 1\right\} \\
& =\min \left\{a_{ \pm}(0)+b_{ \pm}(0), 1\right\} .
\end{aligned}
$$

The following theorem shows that the definition of the sum $\oplus$ is reasonable.

Theorem 4.2 For $A, B, C \in \mathbb{M}[0,1]$ we have

1. $A \oplus B=B \oplus A$;

2. $(A \oplus B) \oplus C=A \oplus(B \oplus C)$.

Proof. Since (1) is obvious by (4.1), we prove here

(2) only. For any $a, b, c \in[0,1]$, we have

(4.2) $\min \{\min \{a+b, 1\}+c, 1\}$

$$
=\min \{a+\min \{b+c, 1\}, 1\} .
$$

Suppose $a+b+c<1$. Then the right as well as left side equals $a+b+c$. Suppose $a+b+c \geqq 1$. Then the right as well as left side equals 1 . The assertion (2) is obvious by (4.2).

\section{Fuzzy Distance}

For $A, B \in \mathfrak{M}[0,1]$ with $A_{\alpha}=\left[a_{-}(\alpha), a_{+}(\alpha)\right]$, $B_{\alpha}=\left[b_{-}(\alpha), b_{+}(\alpha)\right]$ we define an order relation

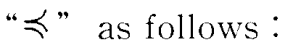

$$
\begin{aligned}
& A \preccurlyeq B \Leftrightarrow \\
& \quad a(\alpha) \leqq b_{-}(\alpha), \quad a_{+}(\alpha) \leqq b_{-}(\alpha), \forall \alpha \in[0,1] .
\end{aligned}
$$

It is easy to see that

$$
\begin{gathered}
A \preccurlyeq B \text { and } B \preccurlyeq A \Rightarrow A=B, \\
0 \preccurlyeq A \text { for all } A \in \mathfrak{M}[0,1],
\end{gathered}
$$

where 0 means the Fuzzy value with the membership function $0(x)$ defined as follows:

$$
0(\mathrm{x})=1 \text { if } x=0 ; \sigma(x)=0 \text { if } x \neq 0 .
$$

Definition 5.1 A map $\widetilde{d}: \mathfrak{M}[0,1]^{2} \rightarrow \mathfrak{M}[0,1]$ is said Fuzzy distance on $\mathfrak{M}[0,1]$ if, for any $A, B, C \in$ $m[0,1]$,

1. $0 \preccurlyeq \widetilde{d}(A, B), \tilde{d}(A, B)=0 \Leftrightarrow A=B$;

2. $\tilde{d}(A, B)=\tilde{d}(B, A)$;

3. $\widetilde{d}(A, B) \preccurlyeq \widetilde{d}(A, C) \oplus \widetilde{d}(C, B)$.

For $A, B \in \mathbb{M}[0,1]$ and $\alpha \in[0,1]$ we put, with the crisp distance $D(A, B)$ in (3.1),

(5.1) $\mathfrak{I}(\alpha)=$

$$
\begin{array}{r}
{\left[\sup _{(1) \beta \leq \alpha} \max \left\{\left|a_{-}(\beta)-b_{-}(\beta)\right|,\left|a_{+}(\beta)-b_{+}(\beta)\right|\right\},\right.} \\
D(A, B)] .
\end{array}
$$

As shown in Theorem 5.1, the family $\{\mathfrak{I}(\alpha) \mid \alpha \in$ $[0,1]\}$ of closed intervals satisfies the conditions $\left(2_{1}\right)-\left(2_{3}\right)$ in Theorem 2.2, hence we define as follows:

(5.2) $\widetilde{D}(A, B)=$ the Fuzzy value determined by the family $\{\mathfrak{I}(\alpha) \mid \alpha \in[0,1]\}$.

We will show later that $\widetilde{D}(A, B)$ is a Fuzzy distance.

Theorem 5.1 The family $\{\mathfrak{D}(\alpha) \mid \alpha \in[0,1]\}$ of interval values satisfies the conditions $\left(2_{1}\right)-\left(2_{3}\right)$ in Theorem 2.2, hence it defines a Fuzzy value.

Proof. Put

$$
\begin{aligned}
l(\alpha)= & (\mathcal{D}(\alpha))_{-} \\
= & \sup _{0 \leq \beta \leq \alpha} \max \left\{\left|a_{-}(\beta)-b_{-}(\beta)\right|,\right. \\
& \left.\left|a_{+}(\beta)-b_{+}(\beta)\right|\right\} .
\end{aligned}
$$

(2) For $0 \leqq \alpha_{1} \leqq \alpha_{2} \leqq 1$, we have 


$$
\begin{aligned}
& \ell\left(\alpha_{1}\right)=\sup _{0 \leq \beta \leq \alpha_{1}} \max \left\{\left|a_{-}(\beta)-b_{-}(\beta)\right|,\right. \\
& \left.\left|a_{+}(\beta)-b_{+}(\beta)\right|\right\} \\
& \leqq \sup _{0 \leq \beta \leq \alpha_{2}} \max \left\{\left|a_{-}(\beta)-b_{-}(\beta)\right|,\right. \\
& \left.\quad\left|a_{+}(\beta)-b_{+}(\beta)\right|\right\} \\
& =\ell\left(\alpha_{2}\right) .
\end{aligned}
$$

$\left(2_{2}\right)$ Let $\alpha \in(0,1]$. For any $\varepsilon>0$ there is a $\beta_{0} \in$ $[0, \alpha]$ such that

$$
\begin{aligned}
\ell(\alpha)<\max \left\{\left|a_{-}\left(\beta_{0}\right)-b_{-}\left(\beta_{0}\right)\right|,\right. \\
\left.\left|a_{+}\left(\beta_{0}\right)-b_{+}\left(\beta_{0}\right)\right|\right\}+\frac{\varepsilon}{2} .
\end{aligned}
$$

(a) When $\beta_{0}<\alpha$, we have for any $\delta$

$$
\left(0<\delta<\alpha-\beta_{0}\right) \text {, }
$$

$$
\begin{aligned}
0 \leqq & \leqq \\
< & \max \{-\ell(\alpha-\delta) \\
& \left.\quad-\max \left\{\mid a_{-}\left(\beta_{0}\right)-b_{-}\left(\beta_{0}\right)-b_{-}\right)|,| a_{+}\left(\beta_{0}\right)|,| a_{+}\left(\beta_{0}\right)-b_{+}\left(\beta_{0}\right) \mid\right\}+\frac{\varepsilon}{2} \\
= & \frac{\varepsilon}{2}<\varepsilon .
\end{aligned}
$$

(b) When $\beta_{0}=\alpha>0$, by the left continuity of $a_{ \pm}(\alpha), b_{ \pm}(\alpha)$ for $\alpha>0$, there is a $\sigma_{0}>0$ such that for any $\delta\left(0<\delta<\sigma_{0}\right)$,

$$
\begin{gathered}
\max \left\{\left|a_{-}(\alpha)-b_{-}(\alpha)\right|,\left|a_{+}(\alpha)-b_{+}(\alpha)\right|\right\} \\
-\max \left\{\left|a_{-}(\alpha-\delta)-b_{-}(\alpha-\delta)\right|,\right. \\
\left.\left|a_{+}(\alpha-\delta)-b_{+}(\alpha-\delta)\right|\right\}<\frac{\varepsilon}{2}
\end{gathered}
$$

hence

$$
\begin{gathered}
0 \leqq \ell(\alpha)-\ell(\alpha-\delta) \\
<\max \left\{\left|a_{-}(\alpha)-b_{-}(\alpha)\right|,\right. \\
\left.\left|a_{+}(\alpha)-b_{+}(\alpha)\right|\right\}+\frac{\varepsilon}{2} \\
-\max \left\{\left|a_{-}(\alpha-\delta)-b_{-}(\alpha-\delta)\right|,\right. \\
\left.\left|a_{+}(\alpha-\delta)-b_{+}(\alpha-\delta)\right|\right\} \\
<\frac{\varepsilon}{2}+\frac{\varepsilon}{2}=\varepsilon .
\end{gathered}
$$

From (a) and (b) we have the left continuity of $\ell(\alpha)$.

$\left(2_{3}\right)$ For any $\varepsilon>0$, by the right continuity of $a_{ \pm}(\alpha), b_{ \pm}(\alpha)$ at $\alpha=0$, there is $a \eta>0$ such that, for any $\gamma \in[0, \eta)$, we have

$$
\begin{gathered}
\max \left\{\left|a_{-}(\gamma)-b_{-}(\gamma)\right|,\left|a_{+}(\gamma)-b_{+}(\gamma)\right|\right\} \\
<\max \left\{\left|a_{-}(0)-b_{-}(0)\right|,\right. \\
\left.\left|a_{+}(0)-b_{+}(0)\right|\right\}+\frac{\varepsilon}{3},
\end{gathered}
$$

hence we have that for any $\gamma \in[0, \eta)$,

$$
\begin{aligned}
\ell(\gamma) \\
=\sup _{0 \leq \delta \leq \gamma} \max \left\{\left|\mathrm{a}_{-}(\delta)-\mathrm{b}_{-}(\delta)\right|,\right. \\
\left.\left|\mathrm{a}_{+}(\delta)-\mathrm{b}_{+}(\delta)\right|\right\}
\end{aligned}
$$

$$
\begin{aligned}
&<\max \left\{\left|a_{-}(0)-b_{-}(0)\right|,\right. \\
&\left.\left|a_{+}(0)-b_{+}(0)\right|\right\}+\frac{\varepsilon}{2},
\end{aligned}
$$

which implies that

$$
\begin{aligned}
& 0 \leqq \ell(\gamma)-\ell(0) \\
& <\max \left\{\left|a_{-}(0)-b_{-}(0)\right|,\right. \\
& \left.\qquad\left|a_{+}(0)-b_{+}(0)\right|\right\}+\frac{\varepsilon}{2} \\
& -\max \left\{\left|a_{-}(0)-b_{-}(0)\right|,\left|a_{+}(0)-b_{+}(0)\right|\right\} \\
& =\frac{\varepsilon}{2}<\varepsilon .
\end{aligned}
$$

Hence $\ell(\alpha)$ is right continuous at $\alpha=0$.

Theorem $5.2 \tilde{D}$ in (5.2) satisfies the conditions (1) $-(3)$ in Def. 5.1 .

Proof. We prove here (3) only, since (1) and (2) are clear.

For $\beta \in[0,1]$ we have

$$
\begin{aligned}
\max & \left\{\left|a_{-}(\beta)-b_{-}(\beta)\right|,\left|a_{+}(\beta)-b_{+}(\beta)\right|\right\} \\
\leqq & \max \left\{\left|a_{-}(\beta)-c_{-}(\beta)\right|,\left|a_{+}(\beta)-c_{+}(\beta)\right|\right\} \\
& +\max \left\{\left|c_{-}(\beta)-b_{-}(\beta)\right|,\left|c_{+}(\beta)-b_{+}(\beta)\right|\right\}
\end{aligned}
$$

By the relation $\max \left\{\left|a_{-}(\beta)-b_{-}(\beta)\right|, \mid a_{+}(\beta)-\right.$ $\left.b_{+}(\beta) \mid\right\} \leqq 1$ we get

$$
\begin{gathered}
\sup _{0 \leq \beta \leq \alpha} \max \left\{\left|a_{-}(\beta)-b_{-}(\beta)\right|,\left|a_{+}(\beta)-b_{+}(\beta)\right|\right\} \\
\leq \min \left\{\operatorname { s u p } _ { 0 \leq \beta \leq \alpha } \operatorname { m a x } \left\{\left|a_{-}(\beta)-c_{-}(\beta)\right|,\right.\right. \\
\left.\left|a_{+}(\beta)-c_{+}(\beta)\right|\right\} . \\
+\sup _{0 \leq \beta \leq \alpha} \max \left\{\left|c_{-}(\beta)-b_{-}(\beta)\right|,\right. \\
\left.\left.\left|c_{+}(\beta)-b_{+}(\beta)\right|\right\}, 1\right\}
\end{gathered}
$$

hence

$$
\left((\widetilde{D}(A, B))_{\alpha}\right)_{-} \leqq\left((\widetilde{D}(A, C) \oplus \widetilde{D}(C, B))_{\alpha}\right)_{-} .
$$

On the other hand, by $D(A, B) \leqq 1$, we get

$$
D(A, B) \leqq \min \{D(A, C)+D(C, B), 1\} .
$$

hence

$$
\left((\widetilde{D}(A, B))_{\alpha}\right)_{+} \leqq\left((\widetilde{D}(A, C) \oplus \widetilde{D}(C, B))_{\alpha}\right)_{+} .
$$

Therefore we obtain

$$
\widetilde{D}(A, B) \preccurlyeq \widetilde{D}(A, C) \oplus \widetilde{D}(C, B),
$$

which proves our theorem.

\section{Conclusion}

In the set $\mathfrak{M}[0,1]$ we have introduced a distance $\widetilde{D}$ which is also a Fuzzy value. For the purpose, we have defined the sum of two Fuzzy values. Further operations on the set $\mathfrak{M}[0,1]$ have been investigated in [1], [3].

Topological properties with respect the Fuzzy distance $\widetilde{D}$ and its applications are topics of further 
research.

\section{References}

[1]Q. Chen, Introductory Analysis of Fuzzy values, Proceedings of the 10th Japan Fuzzy System Symposium, Waseda University, June 4-7 (1996), 239242.

[2]Q. Chen and S. Kawase, Some topological properties of Fuzzy values. To appear in the IEICE Transactions of Fundamentals.

[3]Q. Chen and S. Kawase, Operations and order rela. tions between Fuzzy values. To appear in Fuzzy Sets and Systems.

[4]M. Mukaidono,. Kleene Algebras in Fuzzy Truth
Values, Proceedings of the Fourth International Workshop on Rough Sets, Fuzzy Sets, and Machine Discovery, University of Tokyo, November 68(1996), 37-43.

[問い合わせ先]

Т290-0193

千葉娊市原市潤井户字大谷2289-23

帝京平成大学情報学部情報システム学科

川瀬 奥

TEL. 0436-74-6086

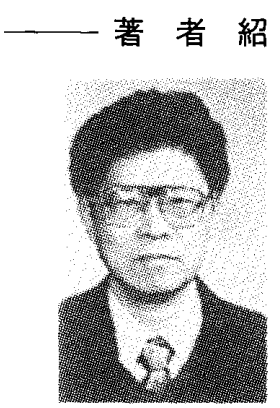

\section{Qihao CHEN}

Beijing University of Post and Telecommunications

Graduated 1964 from the Department of Applied Mathematics, Zhejiang University, China. He is Professor at Beijing University of Post and Telecommunications, and now working at the Department of Math ematics, Chiba University as a Foreign Researcher. His specialities are Fuzzy values, Fuzzy reasoning, etc.

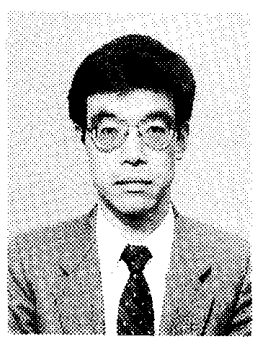

\section{Shin KAWASE}

Teikyo Heisei University

Graduated from Keio University, Faculty of Engineering. Department of Instrumentation at 1963. Obtained Doctor of Engineer degree from Nagoya University at 1984. In 1989-1991, he was Professor in the Department of Administrative Technology at Nagasaki Institute of Applied Science. From 1991, Professor in the Depart. ment of Information Systems at the Teikyou Heisei University.

\title{
Fuzzy Distance between Fuzzy Values
}

\author{
by
}

\section{Qihao CHEN and Shin KAWASE}

\section{Abstract :}

Fuzzy value is a special type of Fuzzy sets on the closed interval $[0,1]$, which is a generalization of triangular type Fuzzy sets. In this note we define, between two Fuzzy values $A, B$, a distance $\widetilde{D}(A, B)$ which is also a Fuzzy value and called a Fuzzy distance. Topological properties with respect to the Fuzzy distance will be investigated in further papers.

Keywords : Fuzzy Sets, Fuzzy Values

\section{Contact Address: Shin KAWASE}

Teikyo Heisei University

2289-23 Uruido Aza Otani, Ichihara-shi, Chiba-ken, 290-0193 JAPAN

TEL : 0436-74-6086 